\title{
La construcción social del calentamiento global cambiando los términos del debate desde américa latina
}

Maribel Guerrero*, José de Souza Silva**

\section{RESUMEN}

Este título es apenas una sugerencia; lo que interesa es su significado. Quiere decir que el calentamiento global es lo que nuestra visión de mundo o concepción de realidad nos permite percibir. En este caso, la humanidad tiene acceso a la naturaleza del calentamiento global y su dinámica a través del discurso oficial hegemónico sobre el tema y, por lo tanto, a través de la visión de mundo de sus protagonistas. Por eso se empieza con "la construcción social" para significar la intervención de valores, intereses y compromisos humanos en el debate sobre el calentamiento global. Sin embargo, se hace el contrapunto estratégico al título, sugiriendo que esta situación es examinada críticamente desde una perspectiva diferente que emerge desde América Latina, distinta de la que prevalece en el debate global.

Palabras clave: Calentamiento global, humanidad, emergente, debate, terminos

\section{ABSTRACT}

This title is just a suggestion, what matters is its meaning. It means that global warming is what our world view or conception of reality allows us to perceive. In this case, humanity has access to the nature of global warming and its dynamics through the hegemonic official discourse on the subject and, therefore, through the world view of its protagonists. So start with "social construction" to mean the involvement of values, human interests and commitments in the debate on global warming. However, it is a strategic counterpoint to the title, suggesting that this situation is critically examined from a different perspective to emerge from Latin America, other than that prevailing in the global debate.

\section{Key words: Global warming, humanity, emergent, debate, terms}

*Universidad Nacional Autónoma de Honduras. Facultad de Ciencias Espaciales. marsury3000@yahoo.com *EMBRAPA para las relaciones ciencia-tecnología-sociedad-innovación (CTSI). Investigador en Sociología de la Ciencia y la Tecnología. Brasil. josedesouzasilva@gmail.com 


\section{INTRODUCCIÓN}

Esta investigación se compone de dos partes: La primera en el campo de la física solar y el clima espacial, donde se analizan las estadísticas obtenidas a partir de datos satelitales de tormentas geomagnéticas, para observar la tendencia del clima en la Tierra hacia un calentamiento o enfriamiento del planeta, como base para rebatir la falsa premisa antropocéntrica de los bien mezclados gases de invernadero (GEI), sobre el mismo discurso que proclama que solamente la idea del forzado natural pueda explicar el calentamiento en los últimos años. Los proponentes del calentamiento global han ignorado intencionalmente recientes investigaciones, que suministran fuertes evidencias científicas de influencia en las relaciones Sol-Tierra reflejadas en la meteorología y el clima.

La segunda parte está inmersa en las Ciencias Sociales para la Gestión del Desarrollo, en la que se analizan las premisas del actual debate sobre el calentamiento global y se postulan las premisas del pensamiento crítico latinoamericano para "La construcción Social del Calentamiento Global".

Existe una relación como un "vínculo orgánico entre el clima y el desarrollo de los pueblos", que afecta las actividades humanas, su productividad, crecimiento económico y que aún no está claro. Ese vínculo orgánico está ligado con transferencia de energía a los seres humanos y hacia los demás seres vivos en general, como la energía magnética, térmica, y luminosa entre otras, por ejemplo, la energía luminosa es la energía que transporta la luz para llevar a cabo la fotosíntesis (capacidad de sintetizar materia orgánica partiendo de la luz y la materia inorgánica). Por otra parte las riquezas naturales como el petróleo, los bosques y fuentes de agua, entre otros, están escaseando por causa de los cambios de clima en forma extrema. Siendo esto materia de controversia para los grupos ecologistas, quienes exageran los daños generados en algunos casos por el aprovechamiento de las riquezas naturales, los grupos cercanos a la industria cuestionan los modelos climáticos computacionales y las predicciones del calentamiento global anunciado.

Así es que podemos darnos cuenta de la necesidad de un cambio de paradigma, que nos prepare para comprender los cambios climáticos como ciclos naturales de nuestro planeta Tierra, tratando de cambiar el paradigma sobre el que descansa el debate actual del calentamiento global, argumentado en premisas paradójicas que presagian un apocalipsis inducido por la misma existencia del ser humano en la Tierra; mostrando una visión del mundo actual sobre este fenómeno, como un cataclismo provocado casi totalmente por los seres humanos. Enfocan la reproducción de la vida humana y el uso de las riquezas naturales como una 
amenaza para la existencia del planeta, principalmente. De distintas maneras lo vemos reflejado en varios informes publicados por el IPCC (siglas en inglés de Panel Internacional de Cambio Climático), estos menosprecian la acción natural del Sol sobre el clima de la Tierra, excluyen las investigaciones científicas del pasado y las emergentes, que contradicen sus premisas y el debate en general.

Este supuesto entró en crisis a partir de la publicación, en noviembre de 2009, de más de un millar de correos electrónicos robados a Phil Jones, -climategate-, director de la Unidad de Investigación del Clima de la Universidad de East Anglia, reconocido científico, influyente miembro del Panel Internacional sobre el Cambio Climático (IPCC), organismo creado por Naciones Unidas a finales de la década de 1980 para enfrentar precisamente el problema del calentamiento global. Lo escrito en esos correos por Jones y algunos de sus colegas lleva a pensar en la manipulación deliberada de datos y en la divulgación de ciertos estudios y rechazo de otros para sostener la mencionada teoría. Jones habla de un ligero enfriamiento desde el 2002 de -0.12 0C en la década, que no debe sorprender, ya que algunos científicos vienen anunciando que debido a la disminución de la actividad solar la temperatura global podría caer por varios años, incluso décadas, en el siglo XXI (Landscheidt, 2003; Njau, 2005; Abdussamatov, 2007; Zhen-Shan y Xian, 2007; Velasco, 2009, 2010) [Armando Páez García 2010]. Existen fuertes cuestionamientos a la teoría del calentamiento como el reporte climate change reconsidered (2009) elaborado por el NIPCC (Panel no gubernamental para el cambio climático).

\section{METODOLOGÍA}

Metodología para la obtención de resultados del impacto de las tormentas solares para los dos últimos mínimos y máximos de actividad solar que causan el calentamiento global o enfriamiento. Se espera determinar por medio de métodos cuantitativos si el calentamiento global es un ciclo natural causado por la actividad del Sol a través de fenómenos coronales, particularmente los que llegan a la Tierra y se convierten en Tormentas geomagnéticas; se estudiarán estos eventos con datos de los "índices K" que miden la actividad geomagnética, para cada día, mes y año la cantidad de fenómenos presentados. Indican la gravedad de las fluctuaciones magnéticas y, por tanto, la perturbación de la ionósfera. Cada observatorio magnético calibra su magnetómetro de modo que su índice K describe el mismo nivel de perturbación magnética cada tres horas, no importa si el observatorio está situado en la regiones aurorales o en el ecuador de la Tierra, con intervalos de tiempo, del tiempo universal (UT). 
Metodología del análisis del debate actual: hacer visibles las verdades invisibles que fundamentan los diferentes discursos sobre el calentamiento global.

Esta metodología facilita el esfuerzo de hacer visibles las premisas invisibles que fundamentan y legitiman los diferentes discursos de los distintos grupos en el debate. Dichas premisas son identificadas a través de las afirmaciones que los diferentes grupos hacen sobre la naturaleza y causas del calentamiento global y su dinámica, porque estas afirmaciones son las premisas a partir de las cuales estos grupos hacen sus promesas y proponen sus soluciones. Para hacer "La Construcción Social del Calentamiento Global" desde América Latina.

\section{RESULTADOS}

Astronómicamente se espera encontrar por medio del análisis estadístico de las tormentas geomagnéticas estudiadas para los años 1989, 1995, 2000, 2006, 2009, 2010 y 2011, observar la actividad solar y la tendencia de su influencia en el clima de la Tierra; inferir que los ciclos de once años están cambiando su comportamiento, correlacionar la actividad solar mínima o máxima con la temperatura sobre la Tierra medida en estaciones meteorológicas y el mar como indicador de la influencia del astro rey, en el último calentamiento global y la variabilidad del clima. Observar y analizar la tendencia de la actividad solar y de tormentas geomagnéticas e inferir el pronóstico del clima de la Tierra hacia un enfriamiento o calentamiento.

\section{CONCLUSIONES}

Con la meta global de un ecosistema planetario sostenible, no excluyente y participativo, que tome en cuenta como la principal fuente natural de energía al Sol y su dinámica propia en la causalidad de los cambios climáticos, debemos reflexionar sobre la necesidad urgente del cambio de paradigma, incluyente en políticas sociales, científicas, educativas, ecológicas y económicas; para comprender los cambios climáticos, su aceptación como ciclos naturales de nuestro planeta ubicado en el SISTEMA SOLAR, en la relación Tierra-Sol y su influencia en el clima, las sociedades del presente y futuro.

La investigación es una síntesis comprensiva, con énfasis en la perspectiva que desde América Latina cuestiona los términos del debate sobre el calentamiento global. La conclusión hace un contraste entre el debate bajo los términos dominantes hoy en el debate global y el debate si éste estuviera bajo la influencia de los términos sugeridos desde América Latina. La conclusión cierra con algunas preguntas, la más relevante de las cuales podría ser la que pregunta sobre las 
implicaciones de continuar el debate bajo los términos hoy dominantes, que están influenciando la naturaleza de los eventos políticos, de las inversiones institucionales, de las iniciativas científicas y del escenario hegemónico emergente. En los discursos e informes oficiales persiste la incertidumbre y muchas contradicciones en probar que solamente el $\mathrm{CO} 2$ es el causante de los cambios climáticos. En defensa de su teoría del calentamiento global antropogénico existe oposición a aceptar que otros factores naturales, como la radiación solar, sea la principal causa, ya que sus efectos no pueden ser controlados por ningún acuerdo internacional.

Políticamente se ha identificado el problema del calentamiento global: el control de los países ricos y más desarrollados sobre el resto del planeta, han instituido el problema en un pequeño círculo económico, se han empoderado del mismo, manipulan su información con falsas premisas, falsas promesas y soluciones inadecuadas.

La investigación propone determinar: Astronómicamente, por medio de análisis estadístico, que el calentamiento global es causado:

- Principalmente por el aumento de la actividad solar, como factor exógeno.

Y que el problema de la variabilidad climática local es causada por:

- El aumento del CO2 por sobrepoblación, tala de bosques, etc., como un factor endógeno menor, comparado con la actividad solar, muy relacionada al contexto local y al impacto del desarrollo humano sostenible.

El impacto de ambos sobre el planeta Tierra, en el transcurso del tiempo; en el aspecto físico, cultural, económico, político y social.

\section{AGRADECIMIENTOS}

A la Dirección de Investigación Científica DICU de la UNAH y su equipo técnico, por el apoyo a mi campo de investigación, dentro de la Astrofísica Solar y las Ciencias Sociales en Gestión para el Desarrollo, que permite el crecimiento profesional de los docentes investigadores y su aporte para un mundo mejor. 


\section{REFERENCIAS}

Álvarez Pomares, O.; Pérez Doval, J. (2002). Solar variability manifestation on earth climate parameters registered at a tropical location. Recuperado de http://adsabs.harvard.edu/abs/2000IAUJD...7E..13A, marzo 2011.

Chen J. (1997). Coronal mass ejections, causes and consequences. En Geophysical Monograph. Florida United States of America, Library of congress cataloging - in publication Data Editorial.

Consecuencias del calentamiento global, Recuperado de

http://ecologismocriollo.blogspot.com/2007/08/muchasorganizaciones-pblicas.html

E. Friis-Christensen and K. Lassen, 1991 Length of the solar cycle: an indicator of solar activity closely associated with climate, Science 254. [Resumen]. Recuperado de

http://www.sciencemag.org/content/254/5032/698.abstract

Elizabeth Peredo. (2010) José Elosegui, Hacia un Tribunal de Justicia Climática,

[Resumen]. Recuperado de http://alainet.org/active/37493.

El calentamiento global en el pasado, 2009, disponible en:

http://www.monografias.com/trabajos61/calentamientoglobal/calentamiento-global2.shtml

Gosling, J.T. et al, 1974, Solar Wind three; proceedings of the Third Conference,

Pacific Grove, LosAngeles, University of California.

Gosling, J.T., 1999, Journal of geophysical research, Volume 104, p. 12515-12524.

Calentamiento global sinopsis, Teorías para explicar el cambio de temperatura,

Recuperado de: http://www.lasnoticiasmexico.com/95636.html.

Howard, R. A et al, 1985, Journal of geophysical research, Vol 90, Recuperado de:

http://www.docstoc.com/docs/58393503/An-Historical-Perspective-to-CME

Hundhausen, A. J, 1996, Coronal mass ejections, in cosmic winds and the heliosphere, eds. J.R. Jokipii et al., Arizona, Tucson.

Hundhausen, A.1999. The many faces of the sun: summary of the results from

NASA's Solar Maximum Mission. Edited by Keith T. Strong, Julia L. et al.

José de Souza Silva, abril del 2001, Publicación mensual del Instituto Científico de Culturas Indígenas. Año 3, No. 25.

José de Souza Silva, 2004 Desarrollo y Dominación Hacia la descolonización del pensamiento subordinado al conocimiento autorizado por el más fuerte.

José de Souza Silva, 2010, Desobediencia epistémica desde Abya Ayala, Pag.1-6, 11-16.

La influencia solar. En BorNet Revista de divulgación sobre ciencia [Artículo].

Recuperado de

http://www.bornet.es/notic/Ciencias_de_la_Tierra_y_Climatologia/ 


\section{1.shtml}

Paez García, Armando, Y después del CO2 ¿Qué?, Mayo 2010, Revista Mad. No 22, pp.1-30.

Sitio Web: SpaceWeather.com, 2007, The Classification of X-ray Solar Flares, disponible en http:// www.Space Weather.com/glossary/flareclasses.html

Sitio Web, Geomagnetic Indices, Octubre 2010, disponible en http://www.swpc.noaa.gov/ftpdir/indices/dayind/README

Sitio web, NOAA Space Weather Scale for Geomagnetic Storms, disponible en http://www.swpc.noaa.gov/ftpdir/lists/geomag/AK.txt

Sitio Web, National weather service, disponible en http://www.swpc.noaa.gov/

ftpmenu/lists/hpi.html

Sitio Web, Geomagnetic Field Data Plot, disponible en http://kogma.nict.go.jp/ cgi-bin/geomaginterface

Sitio Web, Geomagnetic Activity Chart, disponible en http://hirweb.nict.go.jp/ sedoss/geoact

Sitio web Nuestro clima.(2010) Cosas que puedes hacer para frenar el Calentamiento Global. Disponible en

http://blog.nuestroclima.com/calentamiento-global/que-podemos-hacerparafrenar-el-calentamiento-globall

Sitio Web, MAGNE KA and Geomagnetic Data Plot, disponible en

http://hirweb.nict.go.jp/dimages/magneka/19890904t

http://crlhir.nict.go.jp/dimages/magneka/19940220.htm

http://hirweb.nict.go.jp/dimages/magneka/19950101t http://hirweb.nict.go.jp/dimages/magneka/19950801t http://hirweb.nict.go.jp/dimages/magneka/19951111t http://hirweb.nict.go.jp/dimages/magneka/20000101t http://hirweb.nict.go.jp/dimages/magneka/20041208.html http://hirweb.nict.go.jp/dimages/magneka/20060101t http://hirweb.nict.go.jp/dimages/magneka/20060301t http://hirweb.nict.go.jp/dimages/magneka/20061231t Informe Brundtland 2007, [Artículo]. Recuperado de

http://desarrollosostenible.wordpress.com/2006/09/27/informe-brundtland/ http://www.cambioclimatico.redandi.org/node/1247, 2010 http://www.cambioclimatico.redandi.org/node/102, 2009 http://uaim.files.wordpress.com/2010/01/antologia-desarrollo-humano.pdf Serrano, A. (2007). Epistemología de la investigación. En Antología del Doctorado en Ciencias Sociales: VI promoción. Tegucigalpa.

Vázquez-Alonso, A., Manassero-Mas, M. A., Acevedo-Díaz, J. A. y AcevedoRomero, P. (2007). Consensos sobre la naturaleza de la ciencia: La ciencia y la 
tecnología en la sociedad.

[Resumen]. Recuperado de

http://74.125.155.132/scholar?q=cache:AFy_aDLuYIJ:

scholar.google.com $/+\%$ C3\%81ngel+V\%C3\%A1zquezAlonso+et+Al,++200 $7+$ Consensos+sobre+la+naturaleza+de+la+ciencia\&hl=es\&as_sdt=0\&as vis $=1$

Wentzel D. G.( 1989). The restless sun, 1989, Washington D.C. Smithsonian Institution Press. 